\title{
Dissimilaridade entre o estado de humor, humor deprimido e qualidade de vida em atletas com deficiência visual
}

\section{Disimilitud entre el estado de ánimo, el humor deprimido y la calidad de vida en atletas con discapacidad visual}

\section{Dissimilarity among mood state, depressed humor and quality of life in athletes with visual impairment}

\author{
Vigário, P.S. ${ }^{1}$; Lemos, J.R. ${ }^{1}$; Mainenti, M.R.M. ${ }^{2}$; Neves, A.N. ${ }^{2}$ \\ ${ }^{1}$ Centro Universitário Augusto Motta; ${ }^{2}$ Escola de Educação Física do Exército
}

\begin{abstract}
RESUMO
O objetivo foi avaliar o estado de humor, o humor deprimido e a qualidade de vida de atletas de rendimento com deficiência visual, assim como a dissimilaridade entre estes constructos e a prática esportiva. Foi realizado um estudo seccional com 44 atletas com baixa visão ou cegueira total (26,8 $\pm 6,0$ anos de idade; $72,7 \%$ homens), sendo 11 atletas de futebol de cinco, 14 de goalball, 2 de atletismo e 17 de judô. Os seguintes instrumentos na sua versão em português foram utilizados: (i) Profile of Mood States (POMS); (ii) Beck Depression Inventory (BDI) e (iii) Medical Outcomes Study 36-Item Short Form Health Survey (SF-36). Os participantes apresentaram o perfil de iceberg desejável para a POMS, 81,8\% apresentou "nenhum risco" de transtorno depressivo e todos os escores dos domínios do SF-36 estavam acima da média. Foi encontrada correlação positiva entre a duração da sessão de treino e o fator "vigor" da POMS, dentre outras. Os participantes do estudo apresentaram perfil positivo nos estados de humor, baixo risco de ocorrência de transtorno depressivo e uma percepção positiva de qualidade de vida. Parece haver proximidade de elementos da prática esportiva com os estados de humor e domínios da qualidade de vida, e portanto devem ser acompanhados.
\end{abstract}

Palavras-chaves: humor; depressão; qualidade de vida; pessoas com deficiência

\section{RESUMEN}

El objetivo fue evaluar el estado de ánimo, el estado de ánimo deprimido y lacalidad de vida de los atletas com discapacidad visual, así como la disparidad entre estos constructos y la práctica deportiva. Se realizo um estudio transversal con 44 atletas con baja visión o ceguera total (26.8 \pm 6.0 años de edad, $72.7 \%$ hombres): 11 jugadores de fútbol de 5, 14 jugadores de goalball, 2 atletas de atletismo y 17 atletas de judo. Se utilizaron las versions brasileñas de los siguientes instrumentos: Profile of Mood States (POMS); (ii) Beck Depression Inventory (BDI) y (iii) Medical Outcomes Study 36-Item Short Form Health Survey (SF-36). Los participantes presentaron el deseable perfil de iceberg para POMS, el $81.8 \%$ presentó "sin riesgo" de transtorno depresivo y todos los puntajes de los dominios SF36 estuvieron por encima del promedio. Se encontró una correlación positiva entre la duración de la sesión de entrenamiento y el factor "vigor" del POMS, entre otros. Los participantes del estúdio presentaron un perfil positivo en los estados de ánimo, un bajo riesgo de transtorno depresivo y una percepción positiva de la calidad de vida. Parece haber una cercanía entre los elementos de la práctica deportiva con los estados de humor y los dominios de la calidad de vida, y por lo tanto deben acompañarse.

Palabras clave: estado de ánimo; depresión; calidad de vida; personas com discapacidad. 


\title{
Vigário, P.S.; Lemos, J.R.; Mainenti, M.R.M.; Neves, A.N
}

\begin{abstract}
The aim was to evaluate mood, depressed mood and quality of life of athletes with visual impairment, as well as the dissimilarity between these constructs and sports practice. A cross-sectional study was carried out with 44 athletes with low vision or total blindness $(26.8 \pm 6.0$ years of age, $72.7 \% \mathrm{men}): 11$ five-a-side football players, 14 goalball players, 2 athletics and 17 judo athletes. The Portuguese Brazilian versions of the following instruments were used: (i) Profile of Mood States (POMS); (ii) Beck Depression Inventory (BDI) and (iii) Medical Outcomes Study 36-Item Short Form Health Survey (SF-36). Participants presented the desirable iceberg profile for POMS, 81.8\% presented "no risk" of depressive disorder and all scores of SF-36 domains were above average. Positive correlation was found between the duration of the training session and the "vigor" factor of the POMS, among others. The study participants presented a positive profile in mood states, a low risk of depressive disorder and a positive perception of quality of life. There seems to be closeness of elements of sports practice with states of humor and domains of quality of life, and therefore must be accompanied.
\end{abstract}

Keywords: Mood; depression; quality of life; persons with disabilities.

\section{INTRODUÇÃO}

A deficiência visual é uma das mais prevalentes em todo o mundo (Congdon, Friedman, \& Lietman, 2003), com uma estimativa de 285 milhões de casos (World Health Organization, 2010). O Brasil apresenta um comportamento epidemiológico semelhante: os dados do censo de 2010, último para tal abordagem até o momento, apontam que a deficiencia visual é a mais prevalente entre os brasileiros, presente em $23.9 \%$ da população (Instituto Brasileiro de Geografia e Estatística, 2010).

Compreender como o exercício físico e o esporte podem ser benéficos para esta população já foi tema de estudos prévios, nos quais foram geradas evidências a respeito da melhora do controle motor e fino (Houwen, Visscher, Hartman, \& Lemmink, 2007) da capacidade física (Chen \& Lin, 2011), do equilíbrio (Çolak, Bamaç, Aydin, Meriç, \& Ozbek, 2011), da socialização (Movahedi, MojTahedi, \& Farazyani, 2011), autoestima (Qasin, Ravenscroft, \&Sproule, 2014), entre outros desfechos. As pesquisas que exploram especificamente aspectos psicossociais são mais recentes, com sua origem na década de 1980, nos trabalhos pioneiros de Szyman (1980) e Kennedy (1980). Em uma revisão sistemática sobre o tema em atletas Paralímpicos (Jefferies, Gallagher, \& Dunne, 2012), foi observado que os atletas com deficiência visual são o grupo de atletas menos estimado juntos aos demais atletas paralímpicos (Mastro, Burton, Rosendahl, \& Sherrill, 1996). Todavia, a conexão com os outros, o senso de competencia e a percepção de liberdade são elementos essenciais para a motivação intrínseca (Banack, Sabiston \& Bloom, 2011). Noutra perspectiva, os atletas com deficência visual parecem ter equilibrados as orientações ao ego e á tarefa para o cumprimeto de metas (Pensgaard, Roberts, \& Ursin, 1999).

A pressão pela excelência dos resultados, as preocupações com a possibilidade de lesões e dietas, as dificuldades em equilibrar o esporte com compromissos da vida social, a falta de feedback do treinador, a falta de apoio social no treinamento e a competitividade são alguns fatores estressores específicos em contextos desportivos (Mckay, Niven, Lavallee, \&White, 2008) que impactam os atletas em variáveis de natureza cultural, econômica e simbólica (DoValle, 2003). ). A natureza competitiva dos esportes engloba uma ampla gama de emoções, incluindo tristeza e felicidade, raiva, ansiedade, sentimentos de inferioridade, medo, desesperança, orgulho, que influenciam o desempenho dos indivíduos (Jones, 2003). Em suma, pensamentos e sentimentos negativos podem influenciar o treinamento e a competição (Martin, 2017). No esporte para pessoas com deficiência visual o cenário competitivo e a expectativa de vitória se reproduz com intensidade e comprometimento semelhantes ao exporte para pessoas sem deficiência (Howe, 2015).

Fatores psicológicos são cruciais para a adaptação pessoal e social da pessoa com deficiência. Influem para consistência do desempenho do paratleta e para conduzir as tentativas necessárias para controlar pensamentos e emoções antes do desempenho 


\section{Qualidade de vida e estado de humor}

(Rostami \& Mohammadi, 2015). Com o crescimento do esporte para pessoas com deficiência observado nos últimos anos, tem se tornado importante compreender como esses atletas conseguem atingir o ponto ótimo de suas habilidades e capacidades, em uma perspectiva holística (Jefferies, Gallagher, \& Dunne, 2012), considerando a plena integridade física, emocional, psicológica e social do (Brandão \& Agresta, 2008). A geração evidências científicas no tocante à questões psicossociais em atletas com deficiência visual é relevante pois estes indivíduos além de comporem um grupo vulnerável às influências dos fatores relacionados à prática esportiva e treinamento - como os já apresentados até aqui também podem ser influenciados por fatores específicos inerentes à deficiência. A presente pesquisa tem como foco a vertente psicossocial e foram eleitos três parâmetros que podem fortemente influenciar o desempenho esportivo: transtorno depressivo, estado de humor e qualidade de vida.

Transtorno depressivo refere-se às mudanças de humor, nas quais frequentemente a tristeza encontrase presente. Estão incluídos o transtorno distímico, o transtorno depressivo maior e o transtorno depressivo sem outra especificação (Vandenbos, 2010). O humor, por sua vez, pode ser influenciado por fatores de natureza multimensional, como por exemplo, tensão, ira, fadiga, confusão, vigor e depressão (Terry, Lane, Lane \& Keohane, 1999). Os estados de humor são indicadores do excesso de treinamento (alta fadiga e a depressão), refletindo também as alterações emocionais a ele relacionadas: alta tensão, raiva, confusão e baixo vigor (Rolphs, Carvalho, Rotta, \& Krebs, 2004). Por fim, a qualidade de vida versa sobre como as pessoas vivem, sentem e compreendem o diaa-dia (WHO, 1997). Está associada a fatores como saúde, funcionamento físico, satisfação com a vida, sentimento de felicidade e outros. Independentemente da definição adotada, a qualidade de vida ótima é crucial (Daszykowska, 2006).

Frente às possíveis interferências destas variáveis no desempenho esportivo e aos poucos dados quantitativos relativos aos atletas com deficiência visual (Samulski \& Noce, 2002; Dalla Déa et al., 2011; Rostami \& Mohammadi, 2015), o objetivo primário do presente estudo foi descrever o perfil do estado de humor, o humor deprimido e a qualidade de vida de atletas com deficiência visual. Ademais, buscou-se investigar as diferenças dessas variáveis em função do nível competitivo e da modalidade esportiva praticada, testando a hipótese de que haveria diferença em função destas variáveis categóricas. E\#sperava-se que atelas com experiencia internacional e de modalidades coletivas tivessem melhor estado de humor, menor humor deprimido e maior qualidade de vida. Ademais as associações das variáveis em estudo com parametros da prática esportiva foram investigadas, tentando a hipótese de haver correlações positivas com horas e frequencia semanal de treino, tempo de prática esportiva com estado d ehumor e qualidade de vida e correlações negativas daquelas variáveis com humor deprimido. Por fim, a dissimilaridade de todas as variáveis numéricas em estudo foo investigada exploratoriamente, sem hipóteses a priori.

\section{MATERIAL E MÉTODOS}

Foi conduzido um estudo observacional, do tipo seccional, em que participaram indivíduos com deficiência visual. O estudo foi concebido de acordo com a Resolução 466/2012 do Conselho Nacional de Saúde e foi aprovado pelo Comitê de Ética institucional (17691113.1.0000.5235). Todos os participantes assinaram o Termo de Consentimento Livre e Esclarecido, tendo sido feita a leitura para aqueles tinham perda total da visão.

\section{Participantes}

A população alvo deste estudo são atletas com deficiência visual, incluido aqueles com cegueira total e baixa visão. A amostragem foi feita por conveniência e para serem incluídos no estudo os participantes, homens e mulheres, deveriam: a) ter idade igual ou maior que 18 anos e b) praticar a modalidade esportiva há, pelo menos, seis meses, compondo uma amostra por conveniência. Como critério de exclusão foram considerados: a) indivíduos analfabetos ou aqueles incapazes de compreender as questões contidas nos instrumentos utilizados no estudo $\mathrm{e} \quad \mathrm{b})$ não consentimento para a participação no estudo.

Os 44 atletas avaliados tinham idade $=26.8 \pm 6$ anos, $\mathrm{IMC}=25.8 \pm 3.6 \mathrm{~kg} / \mathrm{m}^{2}$ e a maior parte era do sexo masculino $(72.7 \%)$. Do total, eram 11 atletas de futebol de cinco, 14 de goalball, 2 de atletismo e 17 de judô. O tempo médio de prática na modalidade esportiva foi de $5.7 \pm 5$ anos, com frequência semanal de $4.2 \pm 1.1$ vezes e tempo médio de cada sessão de treino de $4 \pm 2.7$ horas. No que se refere às 


\section{Vigário, P.S.; Lemos, J.R.; Mainenti, M.R.M.; Neves, A.N}

modalidades esportivas, foram avaliados 17 judocas, 11 atletas de futebol de cinco, 14 atletas de goalball e 2 de atletismo. Dentre o total de atletas, 29 tinham cegueira total e 35 tinham baixa visão.

\section{Instrumentos}

- Versão em português da Beck Depression Inventory (BDI; Gorestein \& Andrade, 1998) - Contém 21 itens de múltipla escolha que questionam sobre sentimentos como irritabilidade, desesperança, fadiga, perda de peso, diminuição da libido, entre outros. Pontuações entre 0 e 9 são representativas de ausência de sinais e sintomas de depressão; entre 10 e 19 depressão leve; entre 20 a 30, depressão moderada e; acima de 30 significam depressão grave. Na classificação relativa aos sinais e sintomas de depressão leve, existem ainda as subdivisões: entre 10 a 17 , é considerado como disforia; e entre 17 e 19 indica maior proximidade aos estados de depressão, de fato (Beck, Ward, Mendelson, Mock, \& Erbaugh, 1961) . A versão validada no Brasil, manteve a mesma estrutura fatorial original. Para a amostra do presente estudo, o alfa de Cronbach $(\alpha)$ foi igual a .77 .

- Versão em português da Medical Outcomes Study 36 - Item Short Form Health Survey (SF-36; Ciconelli, Ferraz, Santos, Meinão, \& Quaresma, 1999). É um instrumento genérico para avaliação da qualidade de vida relacionada à saúde composto por 36 itens, que compõem 8 domínios: capacidade funcional, aspectos físicos, aspectos sociais, aspectos emocionais, vitalidade, dor, estado geral de saúde e saúde mental. O escore da escala é transformado em escore razão (raw) que varia de 0 a 100 , no qual altos valores indicam maior satisfação com a qualidade de vida. A validação da versão brasileiramanteve a mesma estrutura fatorial que a versão original e a mesma lógica de interpretação. Para a presente amostra, $\alpha$ $=.71$ para o domínio capacidade funcional, $\alpha=.74$ para aspectos físicos, $\alpha=.71$ para aspectos sociais, $\alpha$ $=.94$ para aspectos emocionais, $\alpha=.63$ para vitalidade, $\alpha=.80$ para dor, $\alpha=.57$ para estado geral de saúde e $\alpha=.84$ para saúde mental. Por sua baixa confiabilidade nesta amostra (Nunally, 1978), o domínios vitalidade e estado geral de saúde foram excluídos das análises.

-Versão em português da Profile of Mood States (POMS; Viana, Almeida, \& Santos, 2001). Escala de 63 itens originalmente criada para avaliar os estudos de humor na clínica, que foi adaptada em um versão reduzida de 36 itens por Peluso (2003) para o uso no contextos esportivos, associando-o ao risco de overtraining. A versão em portuguêsmanteve a estrutura fatorial original de 6 fatores: vigor, tensão, depressão, ira, fadiga e confusão,além de seis itens adicionais que constituem $\mathrm{o}$ fator esportivo específico, chamado desajuste do treino (Raglin \& Morgan, 1989). Para a presente amostra, $\alpha=.77$ para tensão, $\alpha=.78$ para depressão, $\alpha=.72$ para ira, $\alpha$ $=.81$ para "fadiga", $\alpha=.70$ para "confusão" e $\alpha=.59$ para desajuste do treino. Por sua baixa confiabilidade nesta amostra, este último fator foi excluído das análises (Nunally, 1978) .

- Dados antropométricos: foram mensurados a massa corporal total em kg usando-se a balança mecânica da marca Filizola, com intervalos de 100gramas. A estatura, em metros, foi avaliada com estadiômetro Filizola com intervalos de 1 milímetro. Todos os indivíduos foram pesados e medidos estando descalços e vestindo roupas leves. A partir das medidas de MCT e estatura foi calculado o Índice de Massa Corporal (IMC; $\left.\mathrm{kg} / \mathrm{m}^{2}\right)$.

- Questionário demográfico: especialmente elaborado para esta pesquisa, no qual os participantes forneceram informações acerca da sua capacidade de visão (se total ou parcial) e da rotina de treinamento.

\section{Procedimento}

Os participantes foram recrutados em clubes e instituições esportivas do Rio de Janeiro, Brasil e tinham experiência em competições a nível regional, nacional e/ou internacional. $\mathrm{O}$ convite foi feito oralmente, juntamente com a explicação dos objetivos da pesquisa. A partir da adesão voluntária ao estudo, aos participantes foram lidas as escalas e respondidas por eles sem interferência do pesquisador. Cada atleta respondeu individualmente os instrumentos, em espaço previamente determinado pelas instituições. O mesmo pesquisador leu os instrumentos - nesta ordem: BDI, SF-36 e POMS - a todos os participantes, para garantir a confiabilidade externa do instrumento. Em seguida, foram perguntadas informações a respeito da deficiência, e da rotina de treinamento. Ao final os atletas foram pesados e foi mensurada altura. Toda a coleta de dados (leitura, preenchimento dos intrumentos, quetsionário demográfico e dados antropométricos) demorou entre 30-35 minutos. 


\section{Qualidade de vida e estado de humor}

\section{Análise estatística}

Para a descrição inicial dos dados foram calculadas a mediana e os valores mínim e máximo. A fim de se obter uma melhor caracterização de cada parte dos constructos em análise, foram observados separadamente os escores dos fatores constituintes dos instrumentos utilizados. Para verificar a normalidade da distribuição dos dados, foi realizado o teste de Shapiro-Wilk. Os escore do BDI, dos fatores tensão e vigor da POMS e dos domínios dor e vitalidade do SF36 não foram aderentes à distribuição normal. Em assim sendo, optou-se pela utilização de testes nãoparamétricos em todas as análises: coeficiente de correlação de Spearman, Mann-Whitney e KruskalWallis.

Para analisar a dissimilaridade entre as variáveis do estudo, foi realizado escalonamento multidimensional, utilizando o modelo ASCAL, extração Ward e normalizando os escores dos fatores, domínios e dados relativos à atividade física pelo $\mathrm{Z}$ escore. Para o julgamento do melhor ajuste foram considerados dois critérios: (1) o coeficiente de determinação (RSQ), indica a proporção dos dados que são escalonados otimamente pelo modelo, sendo aceitáveis valores superiores a .60 e (2) valor de estresse, que é indicativo de qualidade do modelo, onde menores valores indicam melhor ajuste, sendo estresse zero indicativo de aderência perfeita (Hair, Anderson, Tatham \& Black, 2009). O software SPSS 15.0 foi utilizado em todas as análises e o nível de significância estatística adotado foi de $5 \%$.

\section{RESULTADOS}

\section{Descrição Inicial dos dados}

A mediana, amplitude, média e desvio padrão e significância estatística do teste de normalidade de cada umas das variáveis em estudo podem ser vistos na tabela 1 .

Tabela 1. Análise exploratória e significância estatística do teste de normalidade para cada domínio/ fator dos instrumentos utilizados no estudo e variáveis relativas ao treinamento $(n=44)$.

\begin{tabular}{lcccc}
\hline \multicolumn{1}{c}{ Variável } & Md & Mín & Máx & $\boldsymbol{P}^{*}$ \\
\hline BDI & 8 & 0 & 23 & .29 \\
POMS-Tensão & 6 & 0 & 17 & .18 \\
POMS-Depressão & 1 & 0 & 15 & $<.001$ \\
POMS-Ira & 3 & 0 & 15 & .005 \\
POMS-Fadiga & 2 & 0 & 13 & .004 \\
POMS-Vigor & 18 & 10 & 24 & .21 \\
POMS-Confusão & 4 & 0 & 17 & .04 \\
SF-36-Capacidade funcional & 97 & 65 & 100 & $<.001$ \\
SF-36-Aspectos físicos & 100 & 25 & 100 & $<.001$ \\
SF-36-Dor & 62 & 10 & 100 & .20 \\
SF-36-Aspectos emocionais & 100 & 33.33 & 100 & $<.001$ \\
SF-36-Aspectos sociais & 87.5 & 37.50 & 100 & $<.001$ \\
SF-36-Saúde mental & 84 & 40 & 100 & .13 \\
Tempo de prática modalidade (anos) & 5 & 1 & 22 & $<.001$ \\
Frequência de treinamento/ semana (dias) & 5 & 2 & 6 & $<.001$ \\
Horas de treinamento por dia & 2 & 1 & 8 & $<.001$
\end{tabular}

* Teste de Shapiro Wilk.Significânciaestatísticaquando $\mathrm{P}<0,05$; BDI = BeckDepression Inventory; POMS = Profile of Mood States; SF-36

=Medical Outcomes Study 36 - Item Short Form Health Survey;Md = mediana, Mín=valor mínimo; Máx=valor máximo 


\section{Vigário, P.S.; Lemos, J.R.; Mainenti, M.R.M.; Neves, A.N}

Em relação à POMS, os participantes do estudo apresentaram o perfil de iceberg desejável (Terry, 1994), com o fator vigor com o escore mais elevado que os demais fatores de humor negativo. A BDI teve meidana do escore baixa, que se reflete também na avaliação do risco de ocorrência de transtorno depressivo, sendo que $81,8 \%$ dos participantes tinham nenhum risco de ter o transtorno e $18,2 \%$ apresentaram moderado risco. Quanto ao SF-36, observou-se que todos os escores dos domínios analisados estavam acima da pontuação média de cada domínio, indicando maior tendência à melhor percepção de qualidade de vida.

Comparações entre os participantes: nível competitivo e modalidade

Ao compararmos os atletas que participam de competições a nível nacional $(\mathrm{n}=31)$ com aqueles que competem a nível internacional $(\mathrm{n}=13)$, pode-se verificar, por meio do teste de Mann-Whitney, que não houve diferença significativa em todos os fatores e domínios analisados (Tabela 2).

Tabela 2.Comparações dos sinais e sintomas de depressão, estado de humor e qualidade de vida relacionada à saúde entre os participantes do estudo, segundo o nível competitivo e a modalidade esportiva praticada.

\begin{tabular}{lcccc}
\hline \multirow{2}{*}{ Variável } & $\begin{array}{c}\text { Nível competitivo } \\
\text { (nacional x internacional) }\end{array}$ & \multicolumn{2}{c}{$\begin{array}{c}\text { Modalidade esportiva } \\
\text { (judo x goalball x futebol } \\
\text { de cinco x atletismo) }\end{array}$} \\
\cline { 2 - 5 } & & & $P^{*}$ & $P^{* *}$ \\
\hline BDI & 33.00 & .06 & 5.63 & .14 \\
POMS - Tensão & 79.00 & .79 & .76 & .85 \\
POMS - Depressão & 72.00 & .54 & .95 & .81 \\
POMS - Ira & 72.00 & .54 & 1.33 & .72 \\
POMS - Fadiga & 70.50 & .47 & .89 & .83 \\
POMS - Vigor & 63.50 & .29 & 3.34 & .34 \\
POMS - Confusão & 66.00 & .36 & .87 & .83 \\
SF-36 - Capacidade funcional & 71.50 & .98 & .09 & .99 \\
SF-36 - Aspectos físicos & 56.50 & .69 & 3.06 & .38 \\
SF-36 - Dor & 51.00 & .45 & 5.17 & .16 \\
SF-36 - Aspectos emocionais & 51.00 & .34 & 1.26 & .73 \\
SF-36 - Aspectos sociais & 54.50 & .57 & 2.69 & .44 \\
SF-36 - Saúde mental & 62.50 & .59 & 2.44 & .49 \\
\hline
\end{tabular}

\footnotetext{
* Teste de Mann-Whitney para a comparação de atletas que competem a nível nacional versus nível internacional. Significância estatística quando $\mathrm{p}<.05 ; * *$ Teste de KruskallWallis. Significância estatística quando $\mathrm{p}<.05 ; \mathrm{BDI}=$ Beck Depression Inventory; POMS = Profile of Mood States; SF-36 = Medical Outcomes Study 36 - Item Short Form Health Survey
}

O mesmo foi identificado quando as comparações foram feitas considerando as modalidades esportivas, isto é, judô, goalball, futebol de cinco e atletismo (Tabela 2). 


\section{Qualidade de vida e estado de humor}

Correlação entre os domínios e fatores com a prática de esporte

O tempo de prática da modalidade correlacionou-se negativamente com o fator tensão da POMS $(r=-.41$; $p=.02)$, e a duração da sessão de treino correlacionouse positivamente com o fator vigor da POMS $(r=.57$; $p=.002)$. Nenhuma outra correlação estatisticamente significativa foi observada entre os domínios e fatores avaliados com as características da prática esportiva. As correlações entre os domínios e fatores avaliados podem ser observados na Tabela 3.

Aqui, destacam-se as correlações medias a fortes entre o domínio saúde mental do SF-36 com todos os fatores da POMS, a correlação negativa média entre a BDI e o domínio aspectos emocionais do SF-36, a correlação negativa média entre o escore do fator ira e tensão da POMS com o domínio aspectos sociais do SF-36 e a correlação negativa média entre o fator ira da POMS e o domínio dor do SF-36.

Dissimilaridade entre os fatores, domínios e prática esportiva

A partir das características de prática esportiva (tempo de sessão, frequência semanal e tempo de prática) e dos escores dos fatores e domínios dos instrumentos, foram determinadasas distâncias entre as variáveis, calculadas com distância euclidiana ao quadrado a partir dos valores normalizados pelo escore Z. O modelo com melhor aderência apresentou estresse $=.05$ e RSQ $=.84$, indicando uma boa aderência ao modelo e uma proporção de $84 \%$ dos dados escalonados no modelo (Figura 1).

Pôde-se verificar que o tempo de sessão do treino está mais próximo aos fatores tensão, ira e depressão da POMS. Por sua vez, a frequência semanal de treino e o tempo de prática da modalidade são mais próximos do domínio dor do SF-36. Interessante observar nesta amostra que os demais domínios do SF-36 não estão próximos de nenhum elemento da prática esportiva, aparentemente então, tendo pouca influência entre eles.

\section{DISCUSSÃO}

Este estudo buscou descrever o perfil do estado de humor, o humor deprimido e a qualidade de vida de atletas com deficiência visual, além de explorar possíveis diferenças dessas variáveis em função do nível competitivo e da modalidade esportiva praticada, as associações com variáveis relativas à prática esportiva e, por fim, a dissimilaridade de todas as variáveis numéricas em estudo.

A visão é um dos sentidos mais importantes para a funcionalidade dos indivíduos (Pop-Jordanova, Ristova, \& Loleska, 2014). Por isso, a perda, alteração ou mal funcionamento do sistema visual pode gerar dificuldades para a realização de atividades de vida diária, e isso também pode se apresentar no contexto esportivo. Por essa e outras razões, indivíduos com deficiência visual tendem a ficar mais reclusos e têm sua participação social reduzida, o que pode desestabilizar a saúde mental (Pop-Jordanova, Ristova, \& Lojeska, 2014) e impactar negativamente a qualidade de vida (Elsman, van Rens \& van Nispen, 2018).

$\mathrm{Na}$ amostra deste estudo, todavia, o que se observou foi um perfil positivo da qualidade de vida, tendo os domínios avaliados escores acima do valor médio para cada um deles. Este fato pode ser atribuído aos efeitos benéficos da prática esportiva, conforme também observadopor Lima, Gorgatti, \& Dutra (2010) quando uma amostra de 12 indivíduos com deficiência visual que praticavam futebol e atletismo apresentou maiores scores em cada domínio do SF-36 em comparação a 16 congêneres também com deficiência visual, porém sedentários. Embora não tenham investigado especificamente a qualidade de vida,Durson et al. (2015) constataram que a prática de patinação do gelo em um grupo de crianças e jovens com deficiência visual, entre 8 e 16 anos de idade, levou à redução de problemas emocionais e melhoria na qualidade do sono, aspectos estes que em geral também influenciam positivamente a percepção de qualidade de vida. 


\section{Vigário, P.S.; Lemos, J.R.; Mainenti, M.R.M.; Neves, A.N}

Tabela 3. Correlação entre os domínios e fatores dos instrumentos utilizados no estudo

\begin{tabular}{lcccccccccccc}
\hline \multicolumn{1}{c}{ Variável } & $\mathbf{( 1 )}$ & $\mathbf{( 2 )}$ & $\mathbf{( 3 )}$ & $\mathbf{( 4 )}$ & $\mathbf{( 5 )}$ & $\mathbf{( 6 )}$ & $\mathbf{( 7 )}$ & $\mathbf{( 8 )}$ & $\mathbf{( 9 )}$ & $\mathbf{( 1 0 )}$ & $\mathbf{( 1 1 )}$ & $\mathbf{( 1 2 )}$ \\
\hline 1 BDI & - & & & & & & & & & & \\
2 POMS - Tensão & $.49^{* *}$ & - & & & & & & & & \\
3 POMS - Depressão & $.56^{* *}$ & $.46^{* *}$ & - & & & & & & & & \\
4 POMS - Ira & $.51^{* *}$ & $.72^{* *}$ & $.54^{* *}$ & - & & & & & & & \\
5 POMS - Fadiga & $.51^{* *}$ & $.50^{* *}$ & $.49^{* *}$ & $.52^{* *}$ & - & & & & & & \\
6 POMS - Vigor & -.32 & -.05 & $-.38^{* *}$ & .001 & $-.34^{*}$ & - & & & & & \\
7 POMS - Confusão & .48 & $.43^{* *}$ & $.72^{* *}$ & $.48^{* *}$ & $.65^{*}$ & $-.67^{* *}$ & - & & & & \\
8 SF-36 - Capacidade funcional & -.29 & -.14 & -.23 & -.18 & -.32 & $.38^{*}$ & -.20 & - & & & \\
9 SF-36 - Aspectos físicos & -.18 & .01 & -.06 & .14 & -.07 & $.38^{*}$ & -.07 & $.48^{* *}$ & - & & \\
10 SF-36 - Dor & -.17 & -.30 & -.10 & $-.40^{*}$ & -.23 & .07 & -.16 & .08 & .07 & - & \\
11 SF-36 - Aspectos emocionais & -.40 & -.19 & -.22 & -.16 & .10 & -.01 &.- .5 & .11 & .22 & .19 & - \\
12 SF-36 - Aspectos sociais & -.43 & $.47^{* *}$ & -.37 & $-.54^{* *}$ & -.30 & -.07 & -.26 & .08 & -.19 & .21 & .31 \\
13 SF-36 - Saúde mental & $-.61^{* *}$ & -.59 & $-.68^{* *}$ & $-.56^{* *}$ & $-.58^{* *}$ & $.48^{* *}$ & $-.73^{* *}$ & .24 & .16 & .29 & $.65^{* *}$ & $.36^{*}$ \\
\hline BDI = Beck Depression Inventory; POMS $=$ Profile of Mood States; SF-36=Medical Outcomes Study $36-$ Item Short Form Health Survey &
\end{tabular}




\section{Qualidade de vida e estado de humor}

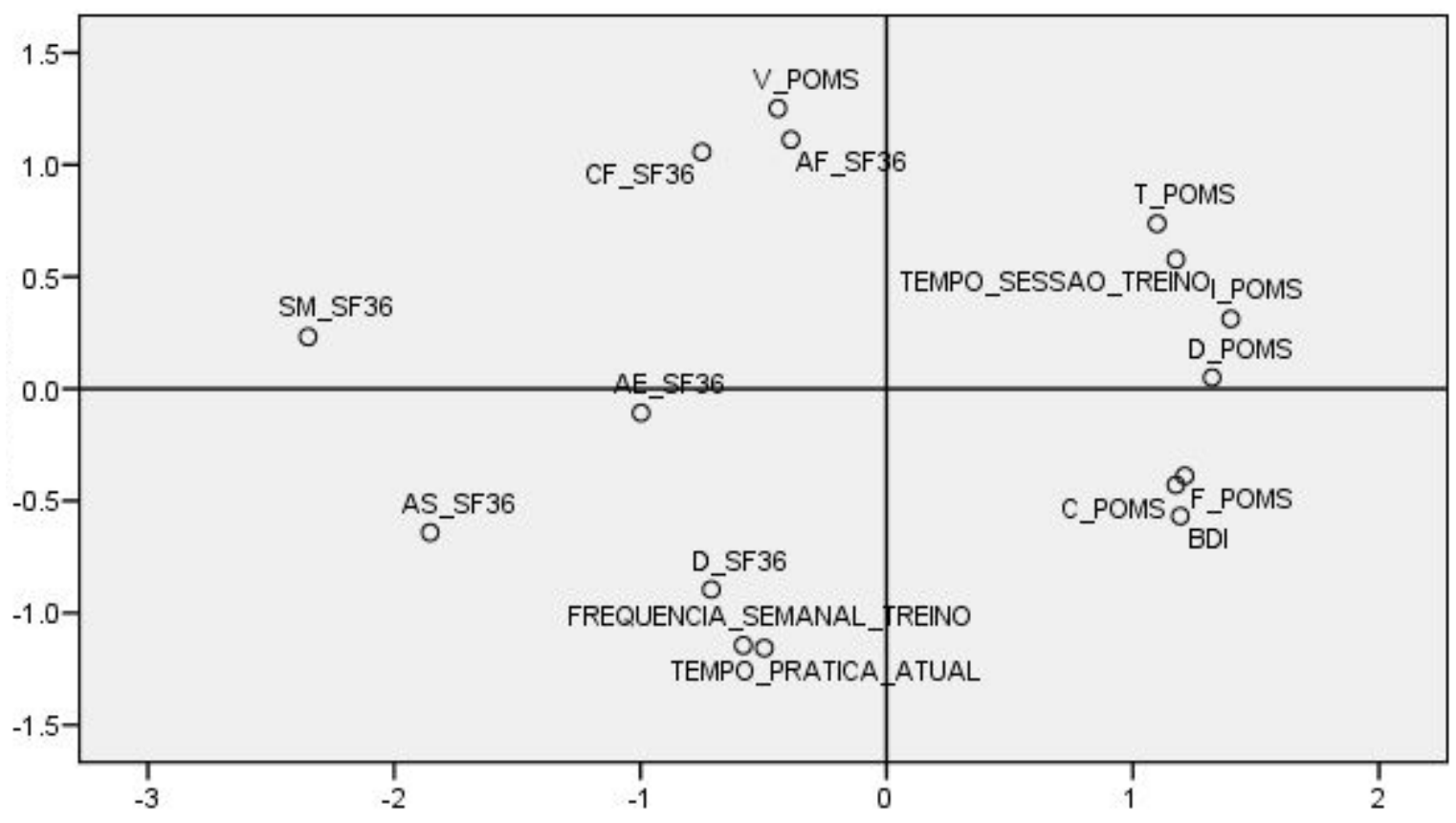

Figura 1.Dissimilaridade entre os fatores da POMS, escore do Inventário de Beck, domínios do SF-36 e prática esportiva.

Legenda: SM_SF36 = Domínio saúde mental do questionário SF-36; AS_SF36 = Domínio aspectos sociais do questionário SF-36; CF SF36 = Domínio capacidade funcional do questionário SF-36; AE_SF36 = Domínio aspectos emocionais do questionário SF-36; D_SF36=Domínio dor do questionário SF-36; V POMS = Fator vigor da POMS; T POMS = Fator tensão da POMS; I_POMS = fator ira da POMS; D_POMS = Fator depressão da POMS; F_POMS = fator fadiga; C_POMS = fator confusão da POMS.

Os resultados apontam para um perfil muito positivo de estado de humor, evidenciando o perfil iceberg desejado (Mastro, Sherill, Gench \& French, 1987). Isso indica que os atletas participantes do estudo estavam fora do risco de supertreinamento, mesmo com alguns treinando até 6 vezes por semana, em sessões que poderiam durar até 8 horas (entre treino tático e técnico). No Brasil, esse mesmo perfil foi observado em outros estudos com atletas com deficiência visual, especificamente nos atletas Paralímpicos de futebol de cinco (Dalla Déa et al., 2011), nos atletas da seleção permanente de atletismo (Rodrigues et al., 2015) e de atletas de goalball com experiência em competições de níveis regional, nacional e internacional (Bonfim, 2017).

Quanto ao risco de haver transtorno depressivo entre os participantes, o mesmo se mostrou pouco evidente, frente aos baixos escores do BDI. Os achados vêm de encontro a estudos prévios sobre depressão em paratletas e depressão em pessoas com deficiência
(Pop-Jordanova, Ristova, \& Loleska, 2014; Khazaeipour, Taheri-Otaghsara, \&Naghdi, 2015; Wolanin, Gross, \& Hong, 2015). Por outro lado, corroboram os achados prévios com atletas da equipe permanente de atletismo Paralímpico de Rodrigues et al. (2015). Apesar da amostra heterogênea do presente estudo -tanto no que diz respeito ao nível competitivo quanto à modalidade praticada - não permitir conclusões sobre uma população específica, os resultados alertam para relevância da prática esportiva nesse público para o equilíbrio do humor.

As duas hipóteses de variancia das variáveis em função da modalidade esportiva e do nível competitivo não foram evidenciadas nesse estudo. E embora os resultados tenham sido majoritariamente positivos, não se deve perder de vista que o ambiente competitivo propicia o surgimento de sinais e sintomas de depressão e ansiedade nos atletas, devido a fatores como afastamento de atividades sociais, de lazer e dos familiares, pressão por resultados e 


\section{Vigário, P.S.; Lemos, J.R.; Mainenti, M.R.M.; Neves, A.N}

treinamento excessivo (Wolanin, Gross, \& Hong, 2015). Com o aumento do desempenho e exposição do esporte Paralímpico Brasileiro, há o aumento da cobrança para os atletas por melhores resultados, e dáse, então, a necessidade de maior suporte da equipe multiprofissional para esses atletas, no acompanhamento detalhado das variáveis emocionais relacionadas à performance esportiva.

Em relação às associações entre as variáveis, salientase a correlação moderada a forte de todos os fatores da POMS - positiva apenas para o fator vigor- com o domínio saúde mental da SF-36. É uma evidencia de que para esta amostra como o humor mais positivo reflete melhor qualidade de vida, no que tange a este domínio. Houve então confirmação parcial da terceira hipótese do estudo.

A dissimilaridade entre as variáveis forneceu insights da influência de três aspectos da prática esportiva tempo de prática na modalidade, sessões semanais e duração da sessão - nas variáveis estudadas. O tempo de sessão de treino tem menor distância em relação aos fatores tensão, ira e depressão da POMS, indicando uma proximidade e maior possibilidade de influência sobre a regulação dos mesmos. Ratificamos que isso não significa que treinos maiores têm efeitos mais positivos - como pudemos ver na ausência de associação entre as variáveis. Já o tempo de prática da modalidade e as sessões semanais se opõem a essa dimensão anterior, formando uma nova dimensão com o domínio aspectos sociais e dor, do SF-36. Mais uma vez, indicamos que estas duas características da prática esportiva têm maior proximidade com estes dois domínios e por sua vez, maior chance de ter influência sobre os mesmos. Não encontramos trabalhos anteriores que tenham realizado esse tipo de análise, por isso, fica impossibilitada a comparação com outros estudos e registrada essa nova perspectiva para a área.

\section{CONCLUSÕES}

$\mathrm{O}$ esporte de alto rendimento praticado por pessoas com deficiência, apesar do seu expressivo crescimento nos últimos anos, ainda busca espaço $\mathrm{e}$ reconhecimento mais consistentes. A carência de estudos envolvendo a área é um fator que colabora para a desinformação da população geral e para o empirismo por parte de alguns profissionais de saúde, visto que ainda há poucos subsídios teóricos que estabeleçam a importância do trabalho e que quantifiquem resultados nesse segmento. . Nesse cenário, apesar das limitações do presente estudo - (a) amostragem por conveniência, que limitam a generalização dos resultados para outras populações; (b) corte transversal, que não permite a verificação de causa-efeito entre as variáveis do estudo; (c) número limitado de variáveis, e (d) desequilíbrio na distribuição dos sexos na amostra - o mesmo buscou dar sua contribuição para o avanço na área. Que seja de conhecimento dos autores, este é primeiro estudo a abordar esta temática em uma amostra de atletas com deficiência visual praticantes de diferentes modalidades esportivas e de diferentes níveis competitivos, e serve como estímulo para o desenvolvimento de outros estudos nesta área de conhecimento.

\section{APLICAÇÕES PRÁCTICAS}

A literatura ainda apresenta lacunas no que diz respeito aos sinais e sintomas de transtorno depressivo, estados de humor e qualidade de vida em atletas com deficiência visual. Sabe-se da importância do acompanhamento biopsicossocial de atletas, levando em conta diversas questões, como a saúde mental, física, aspectos emocionais, culturais, entre outros. Este estudo demonstrou haver proximidade de elementos da prática esportiva com os estados de humor e domínios da qualidade de vida que devem ser acompanhados. Demonstrou também haver, na amostra investigada, um perfil positivo nos estados de humor, baixo risco de ocorrência de transtorno depressivo e uma percepção positiva dos domínios da qualidade de vida. Todavia, frente às exigências de rendimento recomenda-se um acompanhamento multidisciplinar, incluindo profissional da psicologia esportiva, no treinamento destes atletas.

Agradecimento: Coordenação de Aperfeiçoamento de pessoal de Nível Superior (CAPES) e Fundação de Amparo à Pesquisa do Estado do Rio de Janeiro (FAPERJ)

\section{REFERENCIAS}

1. Banack, H. R., Sabiston, C. M., \& Bloom, G. A. (2011). Coach autonomy support, basic need satisfaction, and intrinsic motivation of 


\section{Qualidade de vida e estado de humor}

paralympic athletes. Research quarterly for exercise and sport, 82(4), 722-730.

2. Beck, A. T., Ward, C. H., Mendelson, M., Mock, J., \& Erbaugh, J. (1961). An inventory for measuring depression. Archives of general psychiatry, 4(6), 561571.doi:10.1001/archpsyc.1961.01710120031004

3. Brandão, M. R. F. \& Agresta, M. C. (2008). As Lesões e o Esporte de Rendimento: uma Análise à Luz da Psicologia do Esporte. In.: M. R. F. Brandão, \& A. A.Machado (Eds.).Coleção Psicologia do Esporte e do Exercício: Aspectos psicológicos do rendimento esportivo (Vol. 2) São Paulo,SP: Atheneu

4. Bonfim, B.M.A (2017). Estados de humor $e$ desempenho esportivo de jogadores de goalball. [Dissertação de mestrado em Ciências]. Universidade de São Paulo. Programa de Pósgraduação em Ciências da Atividade Física, São Paulo

5. Chen, C. C., \& Lin, S. Y. (2011). The impact of rope jumping exercise on physical fitness of visually impaired students. Research in Developmental Disabilities, 32(1), 25-29.doi: 10.1016/j.ridd.2010.08.010

6. Ciconelli, R. M., Ferraz, M. B., Santos, W., Meinão, I., \& Quaresma, M. R. (1999). Tradução para o português e validação do questionário genérico de avaliação de qualidade de vida medical outcomes study 36 -item short-form health survey (SF-36) Revista brasileira de reumatologia, 39(3), 143-50.

7. Congdon, N. G., Friedman, D. S., \&Lietman, T. (2003). Important causes of visual impairment in the world today. Jama, 290(15), 2057-2060. doi:10.1001/jama.290.15.2057

8. Çolak, T., Bamaç, B., Aydin, M., Meriç, B., \&Özbek, A. (2004). Physical fitness levels of blind and visually impaired goalball team players. Isokinetics and exercise science, 12(4), 247-252.

9. Dalla Déa, V. H. S., Duarte, E., Gorla, J. I., Inácio, H. L. D., \& Castro, A. P. (2011). Avaliação dos estados de humor dos atletas paraolímpicos brasileiros de futebol de cinco. Pensar a Prática, 14(2), 1-10.
10. Daszykowska, J. (2006). Quality of life in the health-related concepts. The University of Rzeszow Medical Review, 2, 122-128

11. Do Valle, M.P. (2003). Atletas de alto rendimento: identidades em construção. (Dissertação de mestrado). Faculdade de psicologia, Pontifícia Universidade Católica do Rio Grande do Sul, Por Alegre.

12. Dursun, O.B., Erhan, S.E., Ibiş, E.Ö., Esin, I.S., Keleş, S., Şirinkan, A., Yörük, Ö., Acar, E., \& Beyhun, N.E. (2015). The effect of ice skating on psychological well-being and sleep quality of children with visual or hearing impairment. Disability in Rehabilitation. 37(9):783-789. doi: 10.3109/09638288.2014.942002.

13. Elsman, E.B.M., van Rens, G.H.M.B., \& van Nispen, R.M.A.(2018).Quality of life and participation of young adults with a visual impairment aged 18-25 years: comparison with population norms.Acta Ophthalmolica, Sep 11. doi: 10.1111/aos.13903. [Epub ahead of print].

14. Gorenstein, C., \& Andrade, L. H. S. G. (1998). Inventário de depressão de Beck: propriedades psicométricas da versão em português. Revista de Psiquiatria Clinica, 25(5), 245-50.

15. Hair Jr ,J.F., Anderson,R.E.,Tatham, R.L.,\& Black, W.B. (2009). Multivariate Data Analysis. New Jersey: Prentice Hall.

16. Rostami, R., \& Mohammadi, N. (2015). A Comparative Study on Emotional Intelligence and Mental Toughness for Visually Impaired Male and Female Athletes. International Journal of Kinesiology and Sports Science, 3(4), 74-78.

17. Houwen, S., Visscher, C., Hartman, E., \&Lemmink, K. A. (2007). Gross motor skills and sports participation of children with visual impairments. Research Quarterly for Exercise and Sport, $\quad 78(2), \quad$ 16-23.doi: 10.1080/02701367.2007.10599399

18. Howe, P.D. (2015). Disability and Sport: the case of paralympic games. In: R. Giulianotti(Ed). Routledge handbook of the sociology of sport.(p.251-262). Oxon: Routledge.

19. Instituto Brasileiro de Geografia e EstatísticaIBGE. (2010)Censo demográfico 2010: 


\section{Vigário, P.S.; Lemos, J.R.; Mainenti, M.R.M.; Neves, A.N}

Características gerais da população, religião e pessoas com deficiência.Rio de Janeiro: IBGE.

20. Jefferies, P., Gallagher, P., \& Dunne, S. (2012). The Paralympic athlete: a systematic review of the psychosocial literature. Prosthetics and orthotics international, 36(3), 278-289.

21. Jones, M.V. (2003). Controlling emotions in sport. The Sport Psychologist, 17, 471-486

22. Kennedy, M. J. (1980). Spod role socialization and attitudes toward physical activity of wheelchair athletes. Unpublished master's thesis, University of Oregon, Eugene

23. Khazaeipour, Z., Taheri-Otaghsara, S. M., \&Naghdi, M. (2015). Depression following spinal cord injury: its relationship to demographic and socioeconomic indicators. Topics in spinal cord injury rehabilitation, 21(2), 149-155.doi: 10.1310/sci2102-149

24. Lima, C. R.F, Gorgatti, M. G., \& Dutra, M. C. (2010). A influência do esporte na qualidade de vida das pessoas com deficiência visual. Revista Brasileira de Ciências da saúde, 8(23), 40-47

25. Martin, J. J. (2017). Handbook of Disability Sport and Exercise Psychology. Oxford University Press.

26. Mastro, J. V., Sherrill, C., Gench, B., \& French, R. (1987). Psychological characteristics of elite visually impaired athletes: The iceberg profile. Journal of Sport Behavior, 10 (1), 39.

27. Mastro, J. V., Burton, A. W., Rosendahl, M., \& Sherrill, C. (1996). Attitudes of elite athletes with impairments toward one another: A hierarchy of preference. Adapted physical activity quarterly, 13(2), 197-210.

28. McKay, J., Niven, A. G., Lavallee, D., \& White, A. (2008). Sources of strain among elite UK track athletes. The Sport Psychologist, 22(2), 143163.doi: 10.1123/tsp.22.2.143

29. Movahedi, A., Mojtahedi, H., \& Farazyani, F. (2011). Differences in socialization between visually impaired student-athletes and nonathletes. Research in developmental disabilities, 32(1), 58-62.

30. Nunally, J.C. (1978). Psychometric theory. New York: McGraw Hill.
31. Pensgaard, A. M., Roberts, G. C., \& Ursin, H. (1999). Motivational factors and coping strategies of Norwegian Paralympic and Olympic winter sport athletes. Adapted Physical Activity Quarterly, 16(3), 238-250.

32. Peluso,M.A.M.(2003)Alterações de humor associadas à atividade física intensa. [Tese de Doutorado em Medicina]. Universidade de São Paulo. Programa de Pós-graduação em Medicina, São Paulo.

33. Pop-Jordanova, N., Ristova, J., \& Loleska, S. (2014). Depression in ophthalmological patients. Prilozi, 35(2), 53-58.doi: 10.2478/prilozi-2014-0007

34. Qasim, S., Ravenscroft, J., \& Sproule, J. (2014). The Effect of Karate Practice on Self-Esteem in Young Adults with Visual Impairment: A Case Study. Australian Journal of Educational \& Developmental Psychology, 14, 167-185.

35. Raglin, J. S., \& Morgan, W. P. (1980). development Of A Scale To Measure Traininginduced Distress.: 299. Medicine and Science in Sports and Exercise, 21(2), S50.

36. Rodrigues, D. F., Silva, A., Rosa, J. P. P., Ruiz, F. S., Veríssimo, A. W., Winckler, C., ... \& de Mello, M. T. (2015). Sleep quality and psychobiological aspects of Brazilian Paralympic athletes in the London 2012 pre-Paralympics period. Motriz: Revista de Educação Física, 21(2), 168-176. doi: 10.1590/S1980-65742015000200007

37. Rohlfs, I. C. P. D. M., Carvalho, T. D., Rotta, T. M., \&Krebs, R. J. (2004). Aplicação de instrumentos de avaliação de estados de humor na detecção da síndrome do excesso de treinamento. Revista Brasileira de Medicina do Esporte, 10(2), 111-121.

38. Rostami, R., \& Mohammadi, N. (2015). A Comparative Study on Emotional Intelligence and Mental Toughness for Visually Impaired Male and Female Athletes. International Journal of Kinesiology and Sports Science, 3(4), 74-78.

39. Samulski, D., \&Noce, F. (2002). Perfil psicológico de atletas paraolímpicos brasileiros. Revista Brasileira de Medicina do Esporte, 8(4), 157-166. 


\section{Qualidade de vida e estado de humor}

40. Szyman, R. J. (1980). The effect of participation in wheelchair sports. Dissertation Abstracts International, 41, 804A-805A.

41. Terry, P. C., Lane, A. M., Lane, H. J., \& Keohane, L. (1999). Development and validation of a mood measure for adolescents. Journal of sports sciences, $\quad 17(11), \quad 861-872 . \quad$ doi: 10.1080/026404199365425

42. Terry, P. (1995). The efficacy of mood state profiling with elite performers: A review and synthesis. The Sport Psychologist, 9(3), 309324.doi: 10.1123/tsp.9.3.309

43. Vandenbos, G.R. (2010). Dicionário de psicologia da APA. Porto Alegre: Artmed.

44. Viana, M. F., Almeida, P. L. D., \& Santos, R. C. (2001). Adaptação portuguesa da versão reduzida do Perfil de Estados de Humor: POMS. Análise Psicológica, 19(1), 77-92.

45. World Health Organization -WHO (2010). Global data on visual impairments. Disponível em [http://www.who.int/blindness/GLOBALDATAF INALforweb.pdf]. Acesso em 26/13/2017.

46. World Health Organization -WHO (1997). WHOQOL: Measuring Quality of Life. Geneva: WHO (MNH/PSF/97.4)

47. Wolanin, A., Gross, M., \& Hong, E. (2015). Depression in athletes: prevalence and risk factors. Current Sports Medicine Reports, 14(1), 56-60.doi: 10.1249/JSR.0000000000000123 